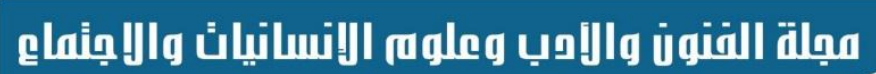 Journal of Arts, Literature, Humanities \\ and Social Sciences
}

ISSN print: 2616- 3810

ISSN online: 2414 - 3383
Volume 48

January 2020
¿ALHSS

www.jalhss.com
(48) - العدد (48)

يناير 2020

\section{القضية الأمازيغية المعاصرة حتى عام 1980م}

\author{
م.د.د. مصعب عطية ذنون الزبيدي \\ كلية التربية الأساسية ـ جامعة سومر - العراق \\ الايميل: mussabateya @gmail.com
}

إن الحديث حول قضايا الأقليات والمكونات الاجتماعية في الدول العربية يعد من القضايا الثائكة، لأن أي حديث في هذا الموضوع هو موقف من قضية حق المكونات والأقليات التي ثتار حولها وحدة وسيادة بلد، لذا يجب على الباحث في مثل هذه المواضيع الالتز ام بالموضو عية والأمانة العلمية والحيادية في عرض هذه القضايا من دون الميول لمكون أو أقلية ما. اذ تكمن صعوبة طرح موضوع النزاع حول قضية الأمازيغ في انتشار هذا المكون في عدة دول عربية افريقية حيث تواجه هذه القضية عدة أنظمة سياسية، وشعور هذه الأنظمة في دول شمال أفريقيا في خطر هذه القضية الحساسة من خلال مطالبتهم بالانفصال أو تهديد كيانهم السياسي أو استغلالهم من قبل قوة إقليمية، دون تفكير هذه الأنظمة في إعطاء حقوقهم وحرياتهم ودمجه في المجتمع من دون اضطهادهم أو سلب حرياتهم، وطيلة فترة الأزمة الأمازيغية لم تمارس هذه الأنظمة سياسة الاحتو اء و الجلوس على طاولة النقاش والاستماع لمطالبهر وتحقيقها أو حتى تحقيق جزء منها، بالعكس مارست جميع أنواع الضغط و التهميش، مما زاد من تعقيد المشكلة والتي استمرت إلى وقتنا الحاضر. لذا تتصب هذه الدر اسة على معرفة هوية وأصول الأمازيخ ونشاطهم السياسي المعاصر ، مرور اً بدخول القضية في عدة أزمات بسبب سياسة أنظمة الدول التي توجد فيها هذا المكون، إذ اتسمت هذه الدراسة بالموضو عية و الحيادية في تناول هذه القضية دون الميول لطرف ما لمعرفة الحقيقة والتعرف على هذا المكون. 


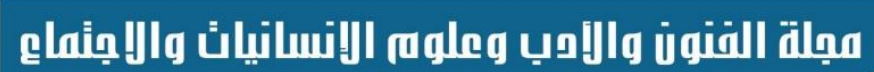 Journal of Arts, Literature, Humanities \\ and Social Sciences \\ ISSN print: 2616- 3810 \\ Volume 48 \\ ISSN online: 2414 - 3383 \\ January 2020 \\ The Tamazight (Berbers) Contemporary Issue until $1980 \mathrm{AD}$
}

\author{
Dr. Musab Atiyah Thanoon Al-Zubaidi \\ Faculty of Basic Education - University of Sumer - Iraq \\ Email: mussabateya@gmail.com
}

\begin{abstract}
Talking about the issues of minorities and social components in the Arab countries is one of the thorny issues, because any conversation in this matter is a position on the issue of the right of the components and minorities that arise around the unity and sovereignty of a country, so the researcher on such issues must adhere to the objectivity and scientific and impartial honesty in presenting These issues are without inclinations of a component or a minority.

As the difficulty of raising the issue of the dispute over the Tamazight issue lies in the spread of this component in several Arab African countries where this issue faces several political regimes, and the feeling of these regimes in North African countries is in danger of this sensitive issue by calling them to secede or threaten their political entity or their exploitation by A regional power, without thinking of these regimes in giving their rights and freedoms and integrating them into society without oppressing them or stealing their freedoms, and throughout the period of The Tamazight crisis these regimes did not practice containment policy and sit at the table of discussion and listen to their demands and achieve them or even achieve part of it, on the contrary I practiced all kinds of Cover and marginalization, increasing the complexity of the problem, which continued to the present. Therefore, this study focuses on knowing the identity and origins of the Berbers and their contemporary political activity, through the entry of the issue into several crises due to the policy of the systems of the countries in which this component exists, as this study was characterized by objectivity and impartiality in dealing with this issue without inclination for a party to know the truth and get to know this component .
\end{abstract}

Keywords: The Tamazight, Africa , the minorities , origins , component. 


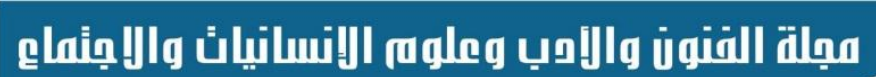 Journal of Arts, Literature, Humanities \\ and Social Sciences}

ISSN print: 2616- 3810

ISSN online: 2414 - 3383
Volume 48

January 2020
¿ÁLHSS

www.jalhss.com

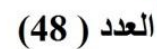

يناير 2020

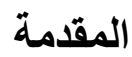

يتز ايد الاهتمام في هذه المدة بقضايا المكونات والاقليات الاجتماعية و الثقافية التي يتكون منها مجتمعاتنا

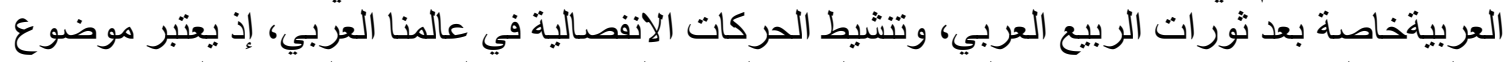

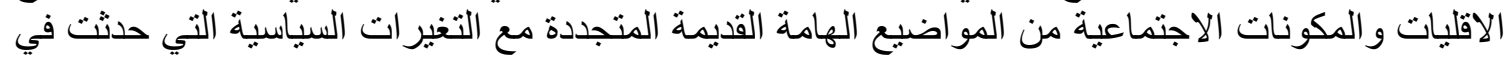

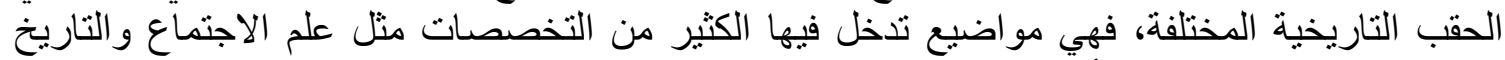

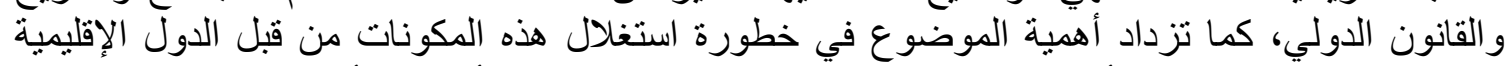

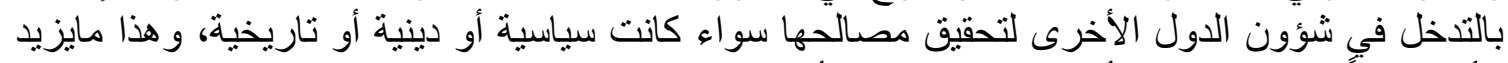
الأمر توتراً مما قد بحول الألمر الألى الى حرب تكون أطر افها دولية.

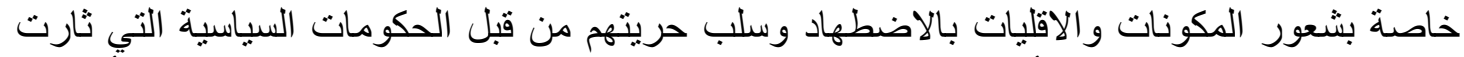

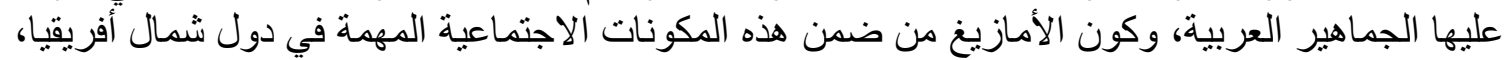

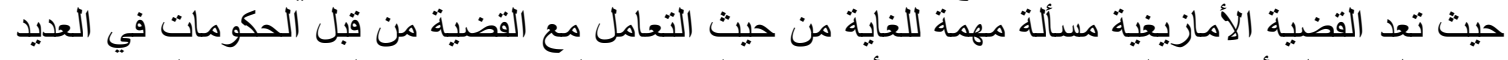

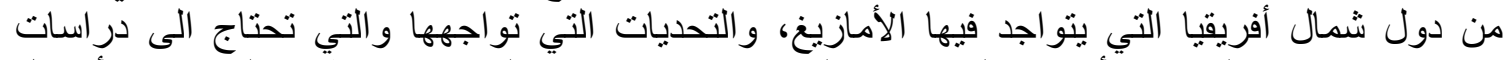

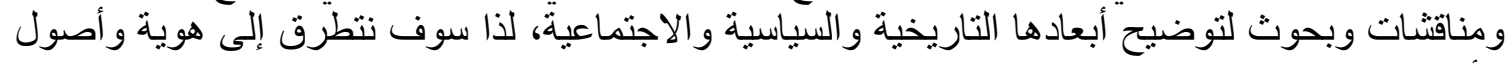

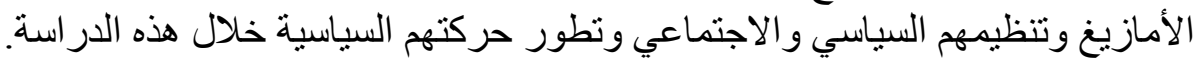

\section{الجذور التاريخية للأمازيغ الماني}

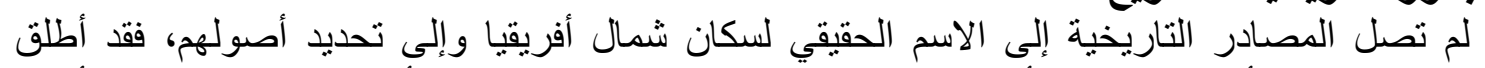

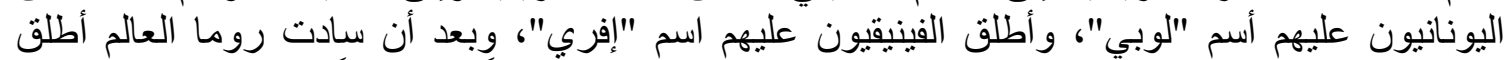

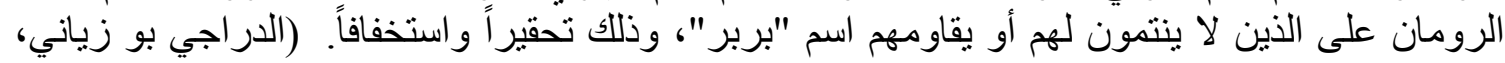

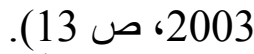

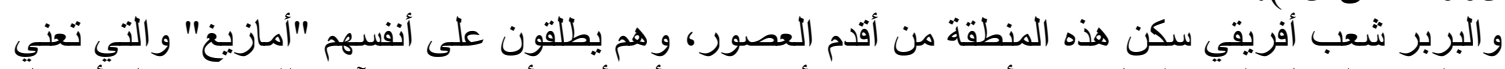

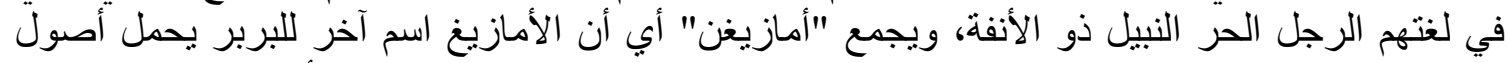

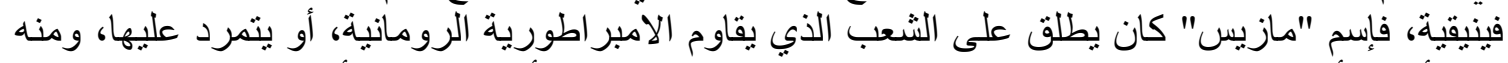

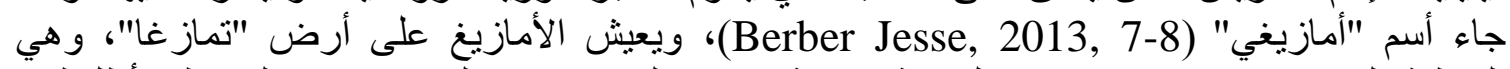

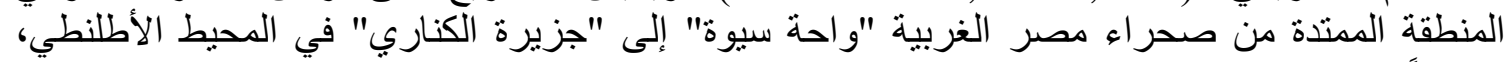

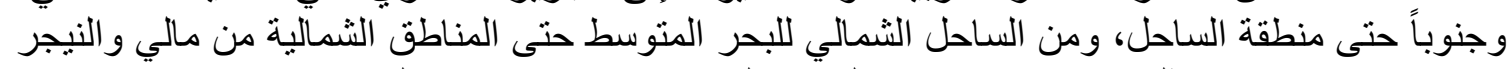

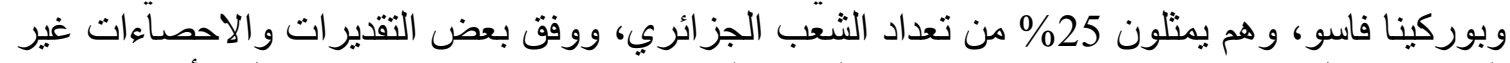

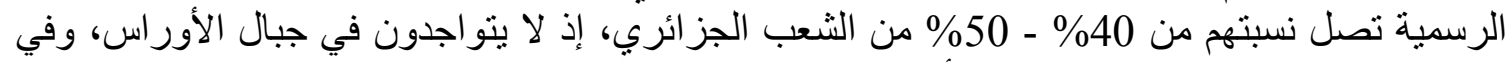

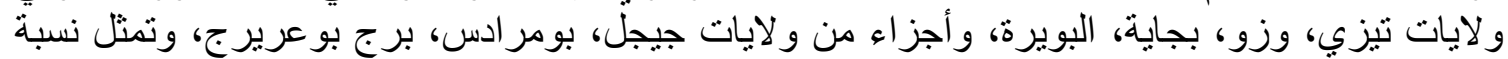

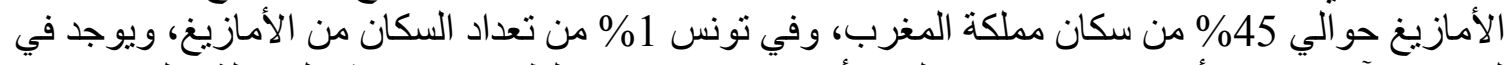

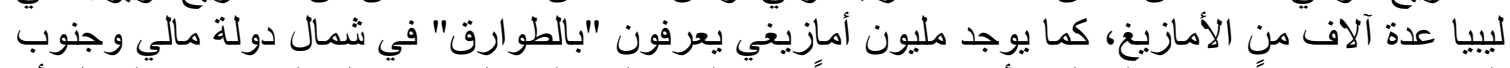

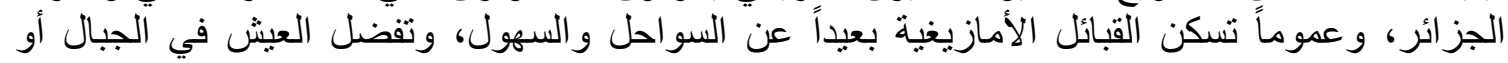

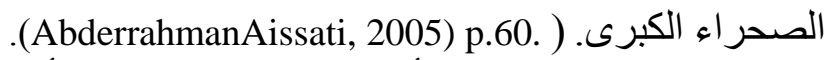

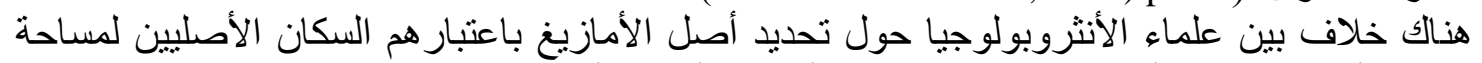

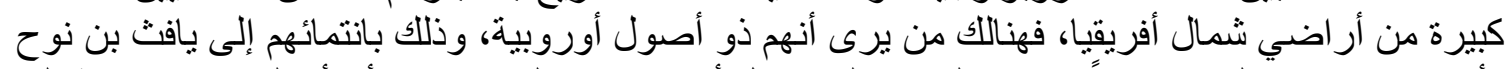

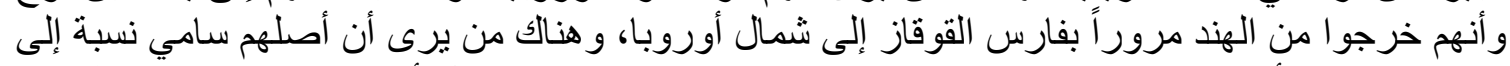

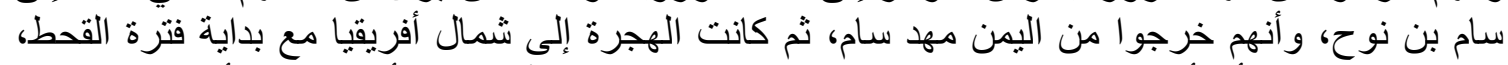

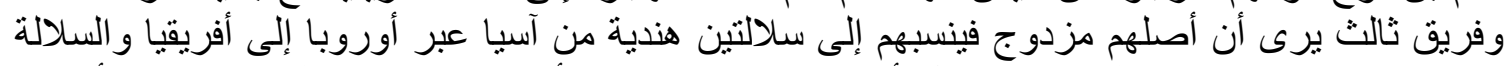

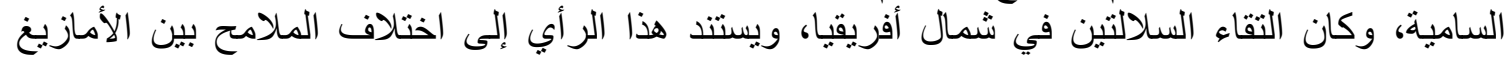




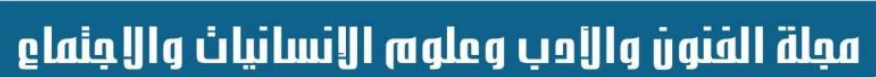 Journal of Arts, Literature, Humanities \\ and Social Sciences}

ISSN print: 2616- 3810

ISSN online: 2414 - 3383
Volume 48

January 2020
LALLHSS

www.jalhss.com

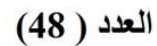

يناير 2020

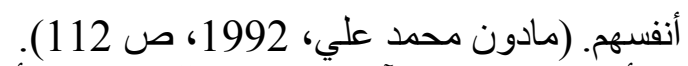

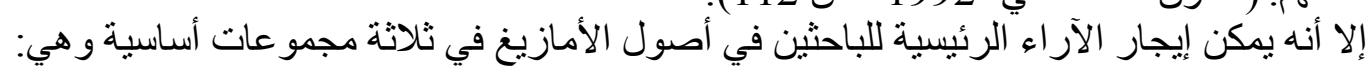

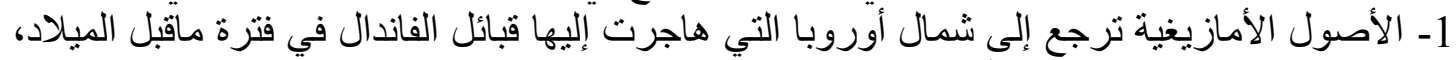

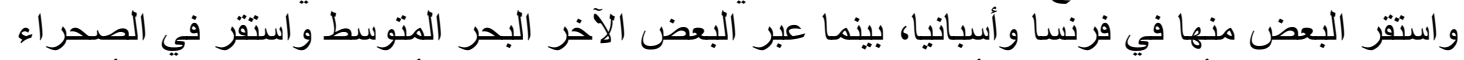

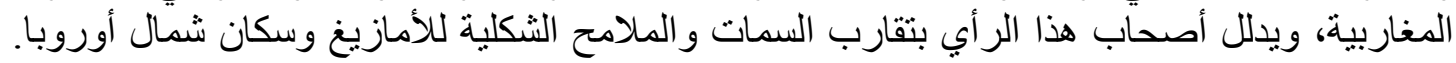
(Maya Shatzmiller, Pubishers, 2000) p.20.) 2- للأمازيغ أصول عربية، إذ يرى البعض أنهم كنعانيين تم طردهم في فلسطين بعد أن قتل النبي داوود

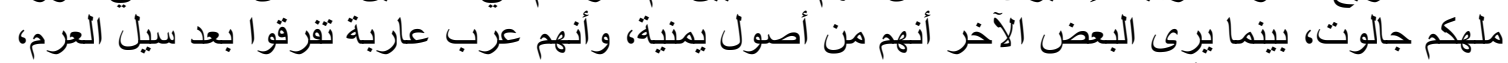

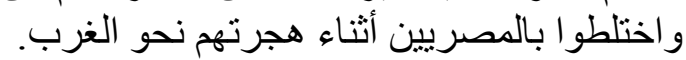
3- الأمازيغ هي شعوب قالمبن قديمة سكنت الصحر اء الغربية منذ آلاف السنين، وأنهم لم يهاجروا إليها لا من

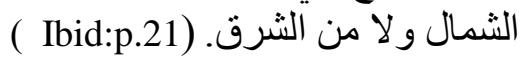
ينقسم الأمازيغ إلى مجمو عات كبرى الثرى تعيش في شمال أفريقيا في أماكن مختلفة يمكن الإشارة إليها فيما يلي:

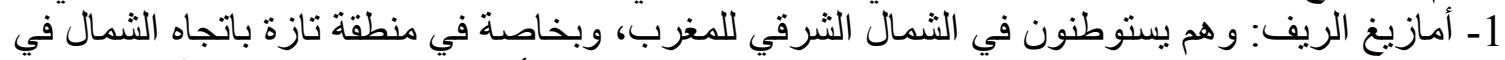

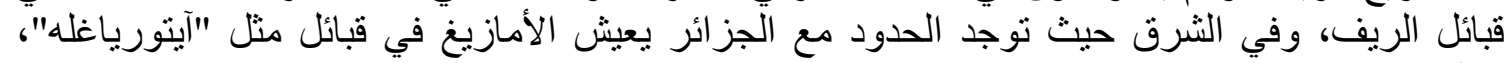

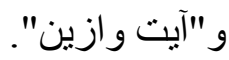

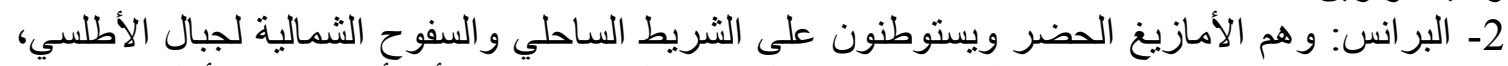

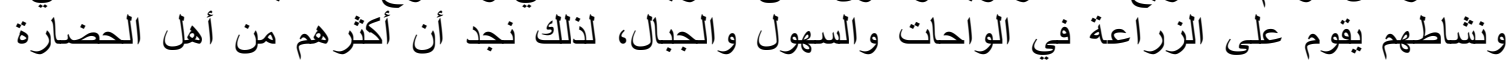

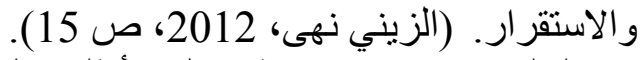
2- الثلوح: وهم في وسط جبال الأني، الأطلس الكبير وغربه وفي منطقة سوس في المغرب، ونستخدم لهجة

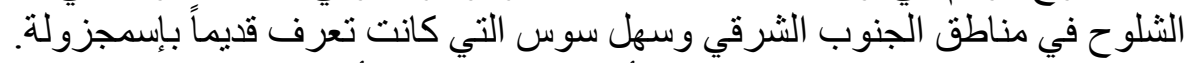

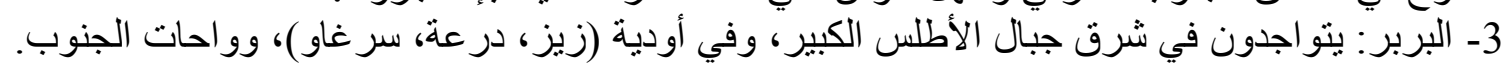

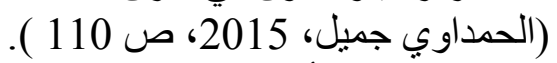

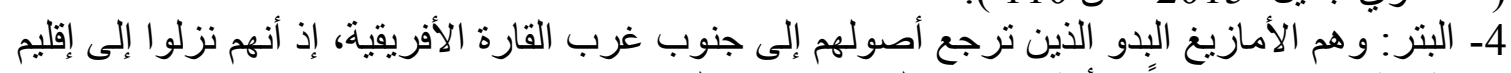

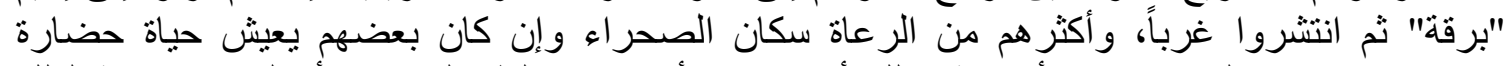

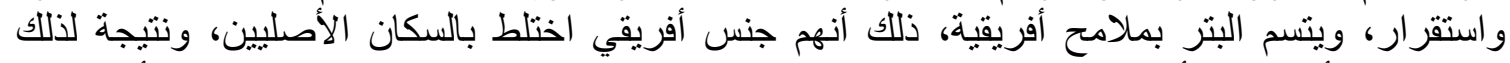

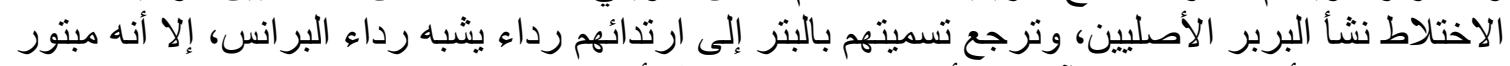

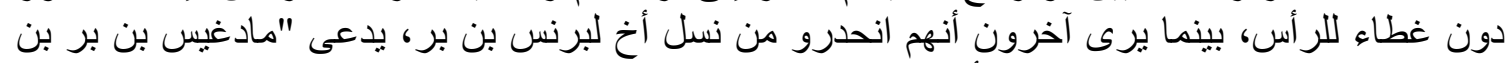

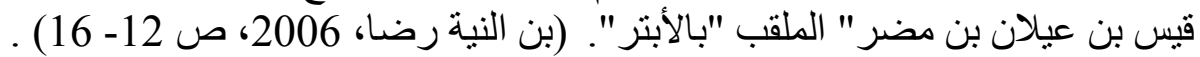

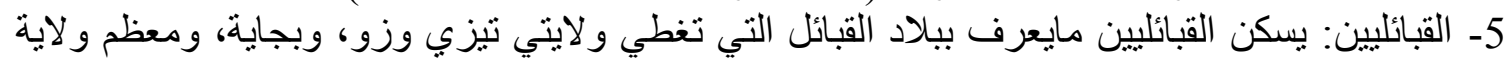

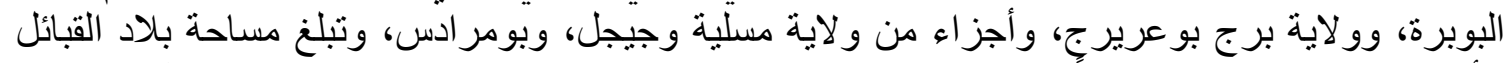

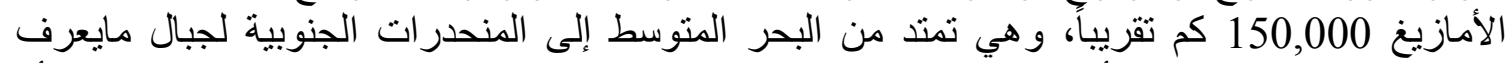

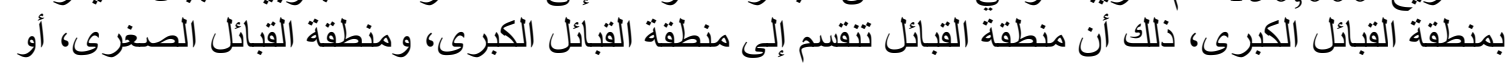

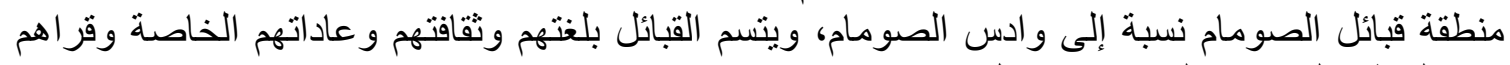

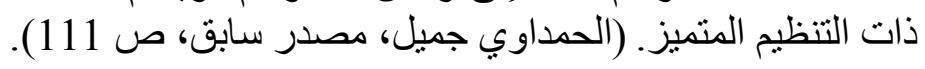

اللغة الأمازيغية

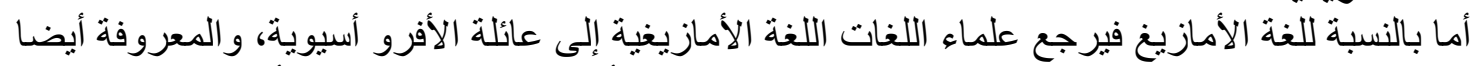

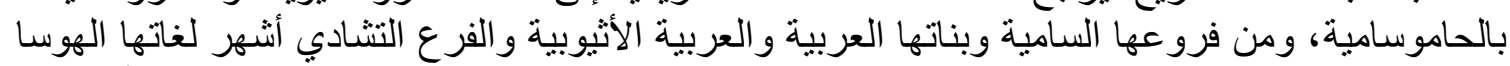
و الفرع الكوشي ومن لغاته الصومالية و الفرع المصري ونية وتطوره في اللغة القبطية في حين تكون الأمازيغية 


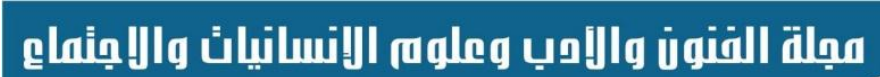 Journal of Arts, Literature, Humanities \\ and Social Sciences}

ISSN print: 2616- 3810

ISSN online: 2414 - 3383
Volume 48

January 2020
¿ÁLHSS www.jalhss.com

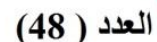

يناير 2020

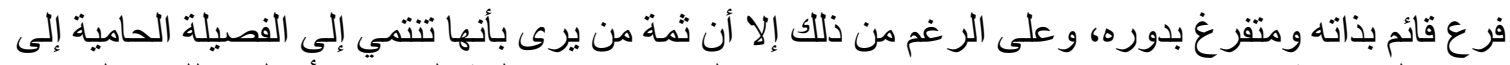

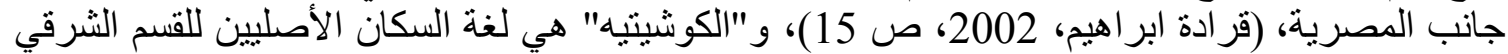

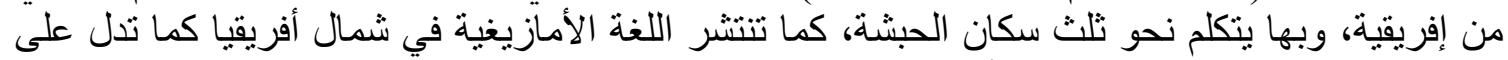

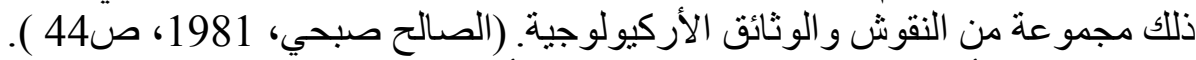

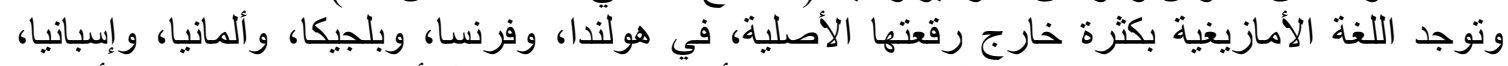

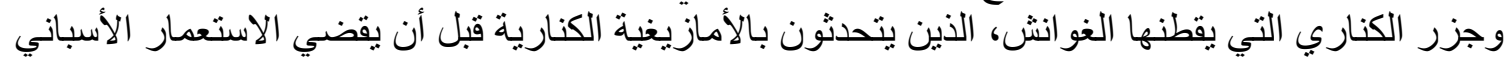

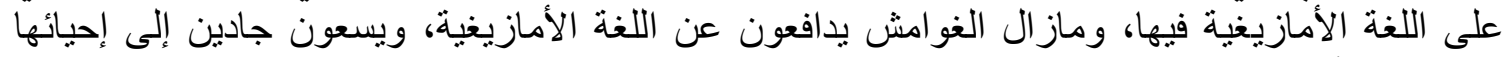

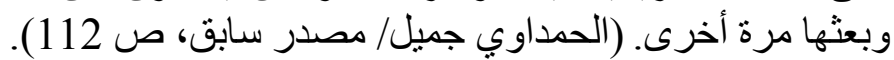

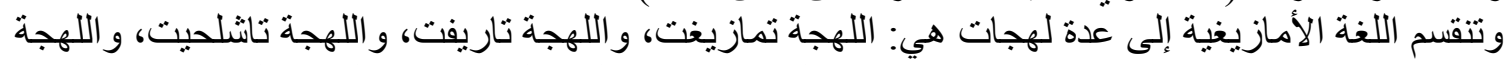

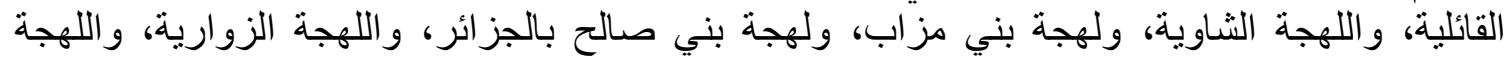

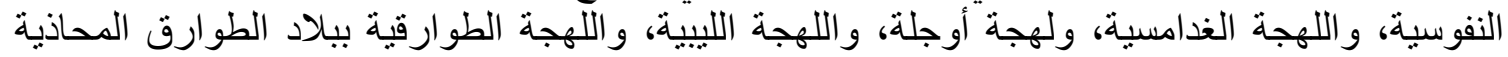

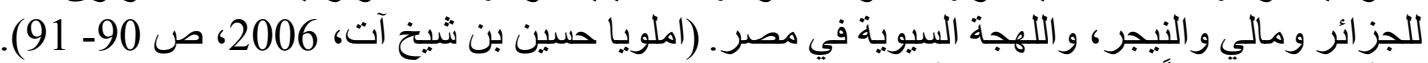

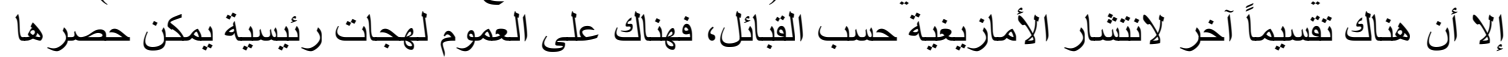

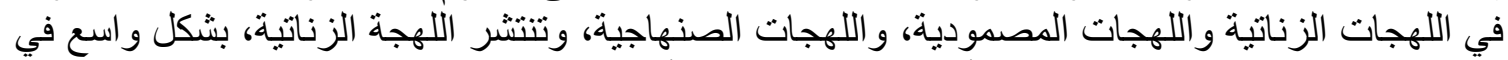

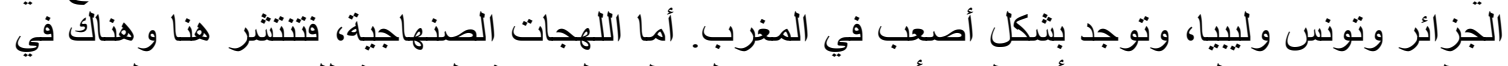

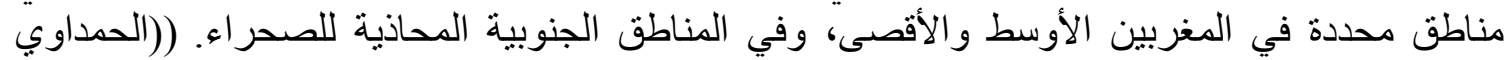

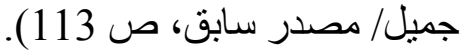

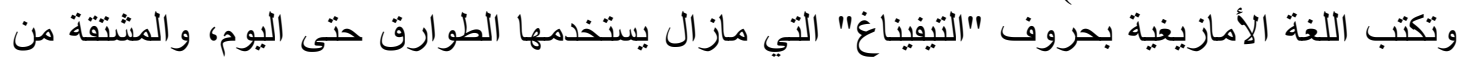

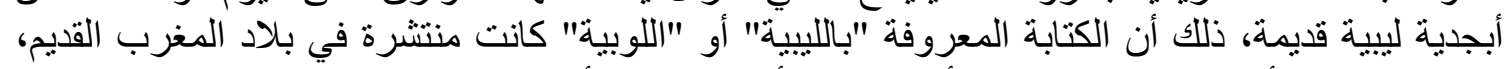

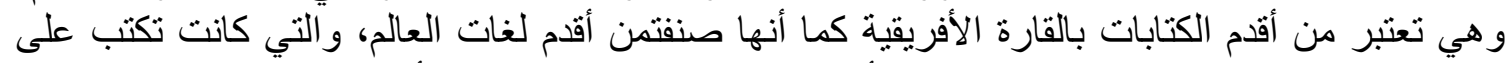

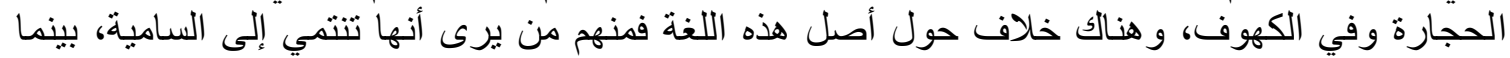

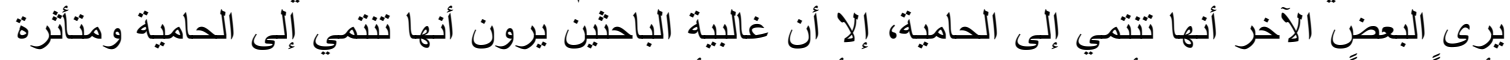

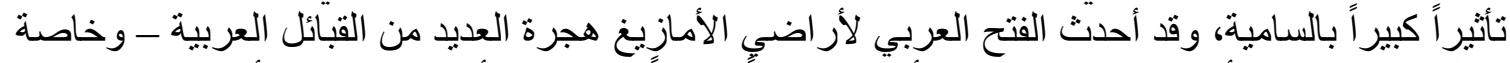

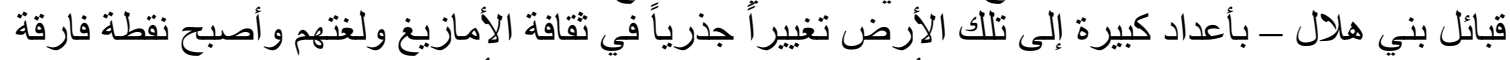

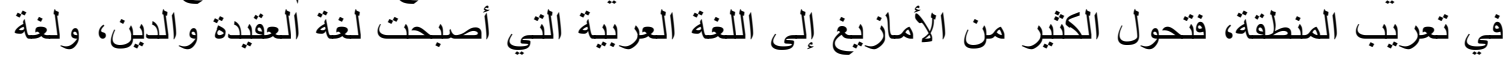

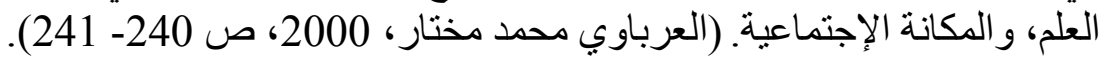

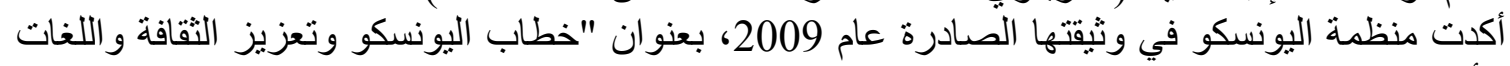

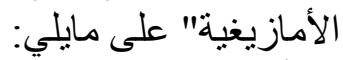
1- أن اللغة والثقافة هما عنصر ان موضية التحان لهوية شعب ذو نركيبة عرقية معقدة.

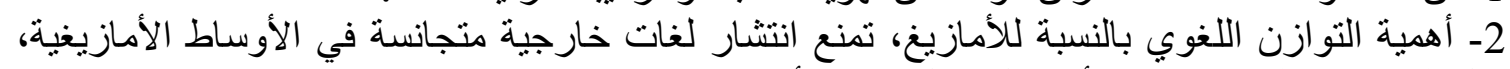

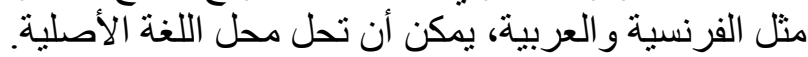

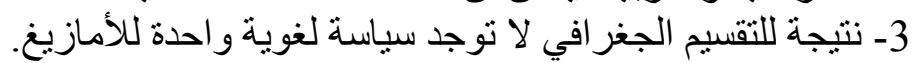

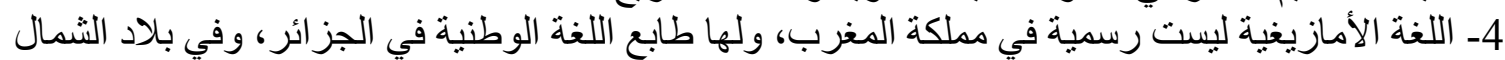

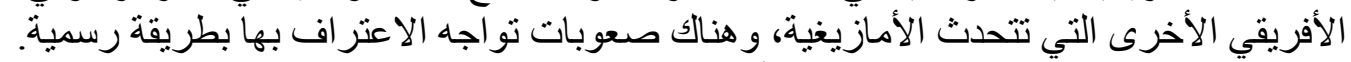

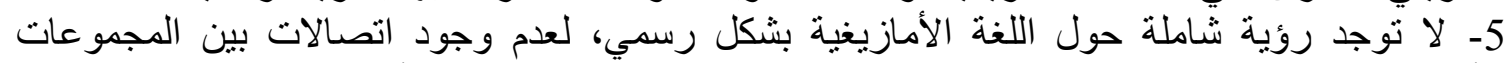

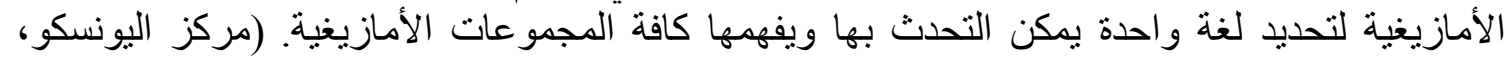

2009، صن 41:)

ومن الجدير بالذكر أن اللغة الأمازيغية هي لغة تخاطب شفهية إذ أن اللهجات الأمازيغية هي لهجات 


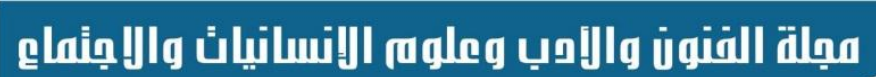 Journal of Arts, Literature, Humanities \\ and Social Sciences}

ISSN print: 2616- 3810

ISSN online: 2414 - 3383
Volume 48

January 2020
¿ÁLHSS

www.jalhss.com

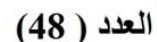

يناير 2020

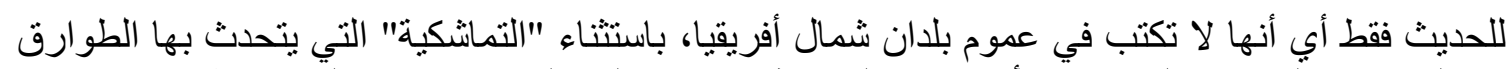

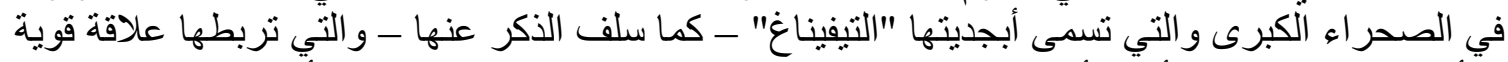

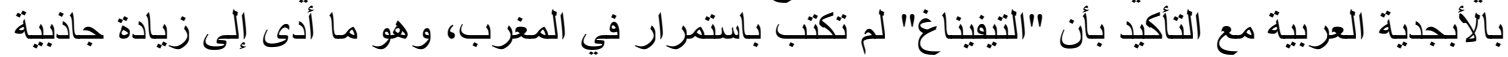

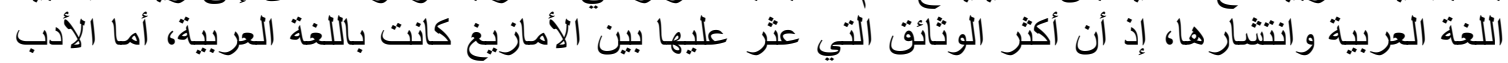

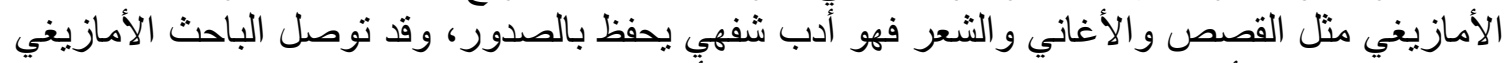

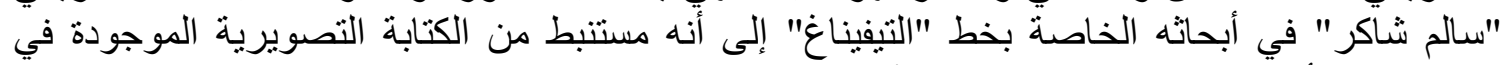

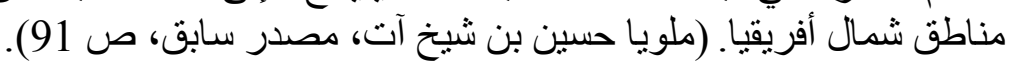

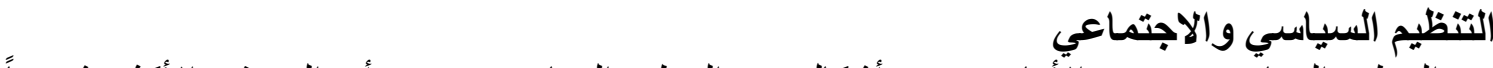

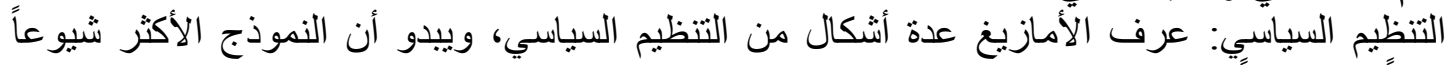

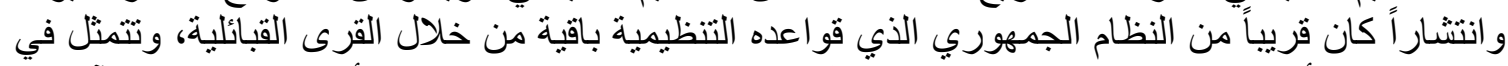

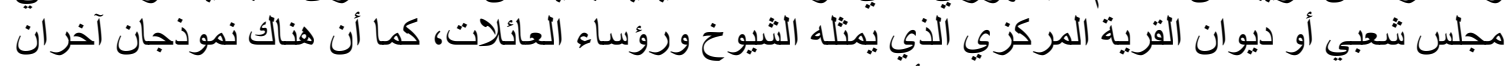

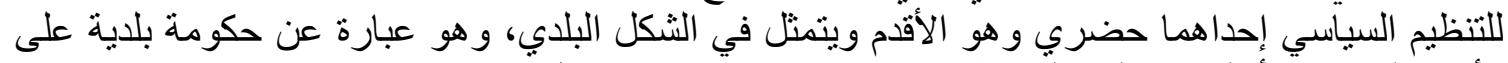

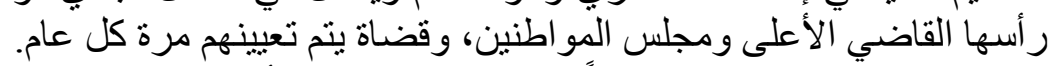

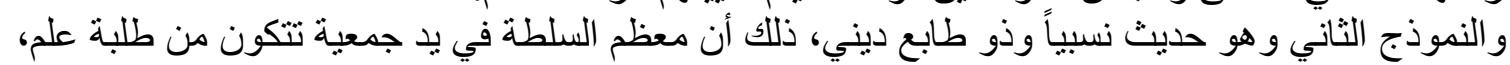

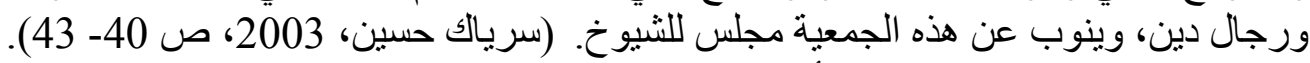

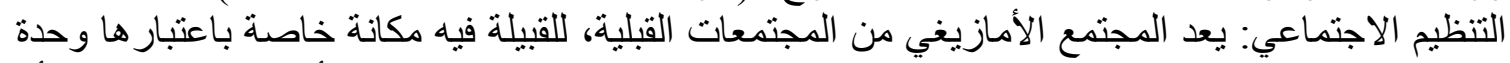

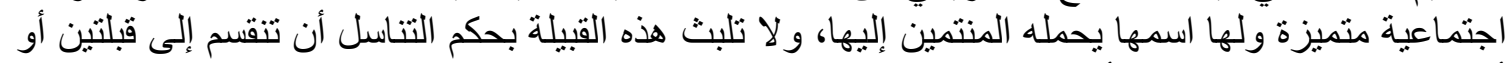

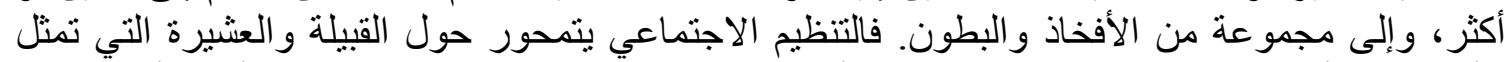

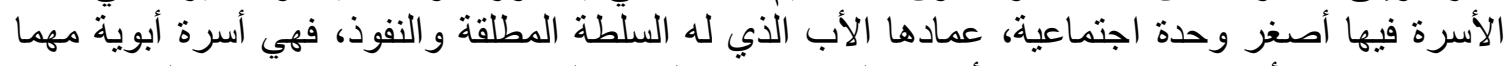

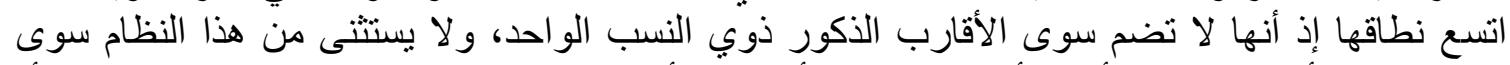

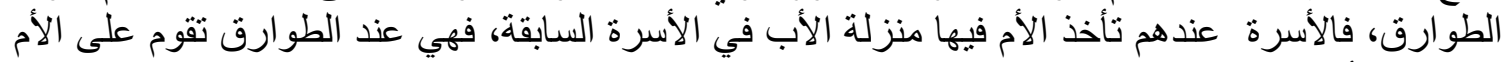

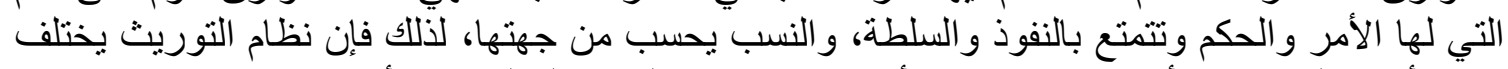

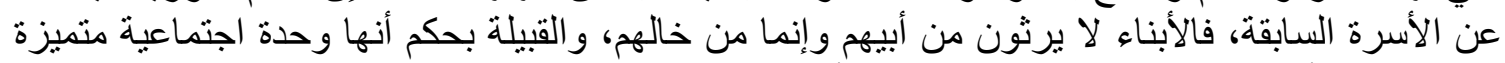

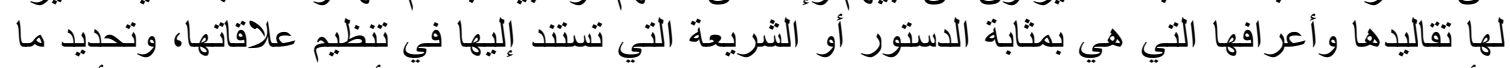

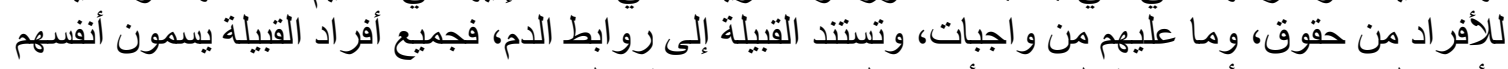

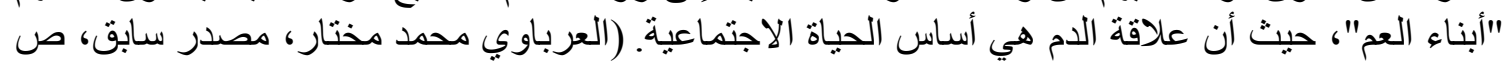
. (368

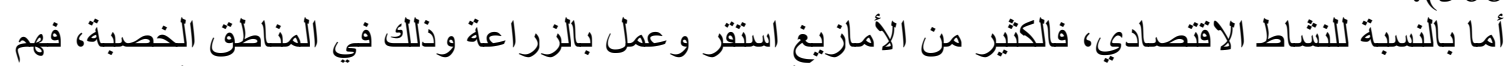

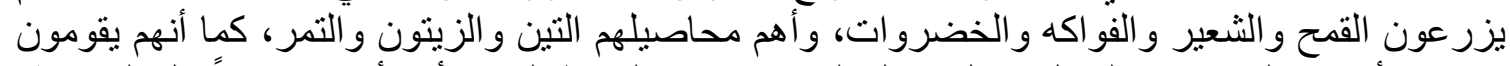

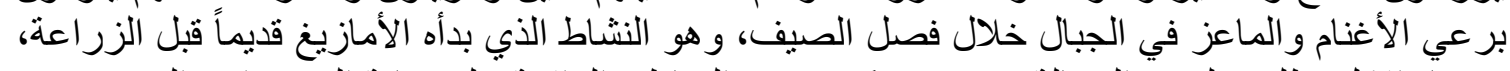

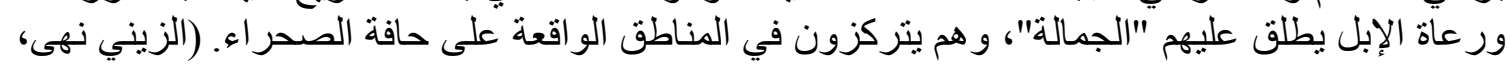

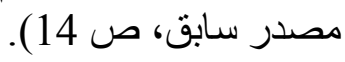

تطور ظهور الحركة الأمازيغية

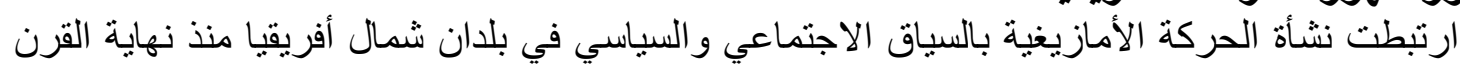

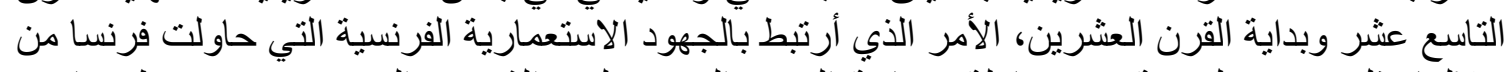

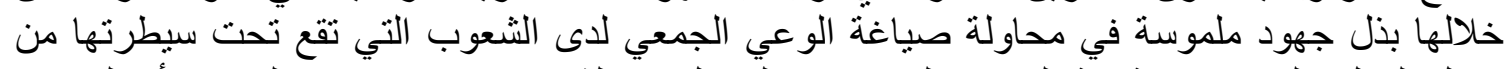

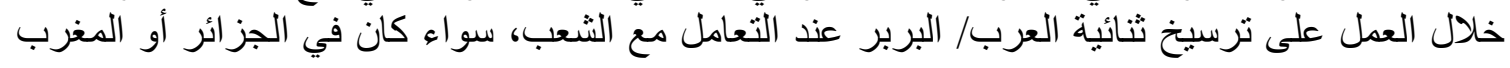




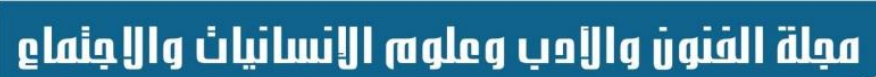 Journal of Arts, Literature, Humanities \\ and Social Sciences}

ISSN print: 2616- 3810

ISSN online: 2414 - 3383
Volume 48

January 2020
¿ÁLHSS www.jalhss.com

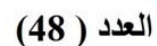

يناير 2020

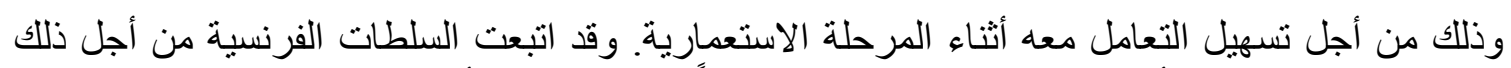

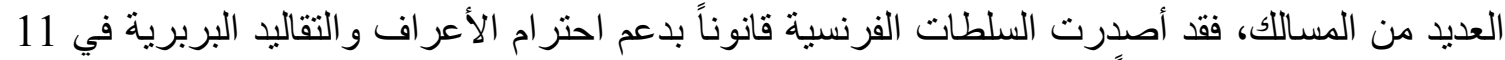

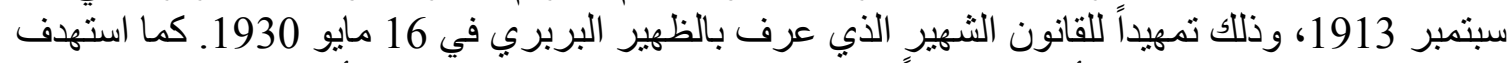

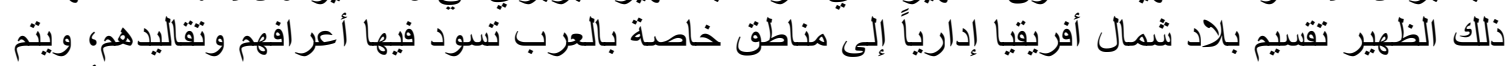

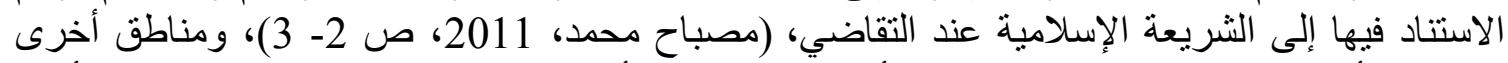

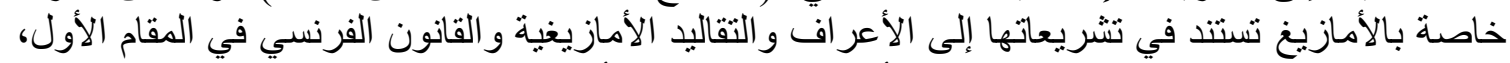

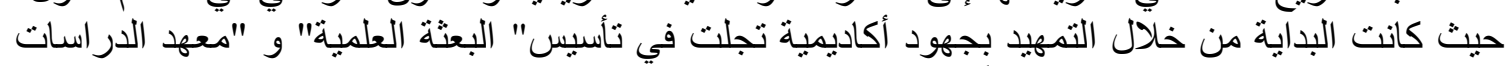

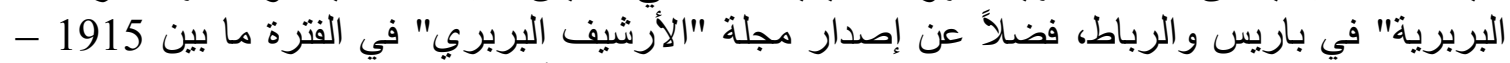

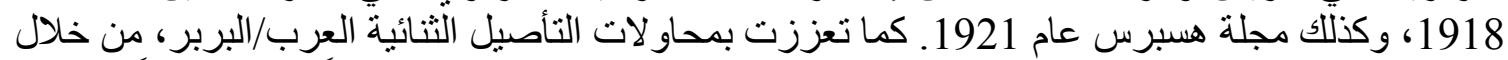

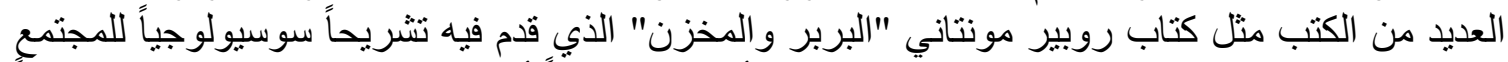

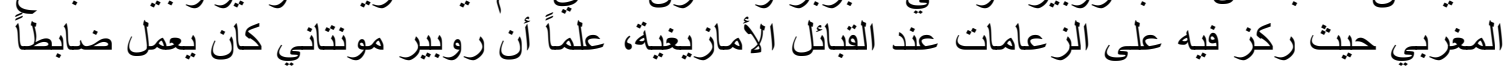

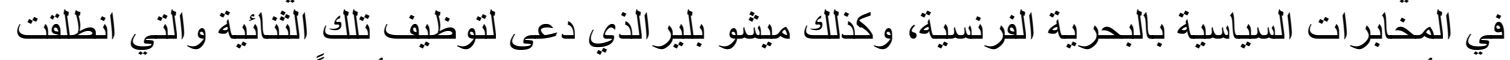

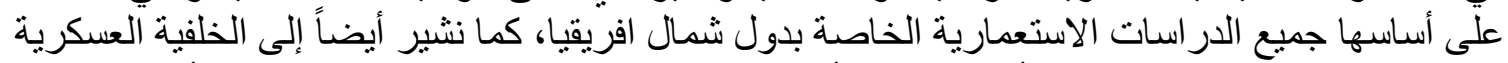

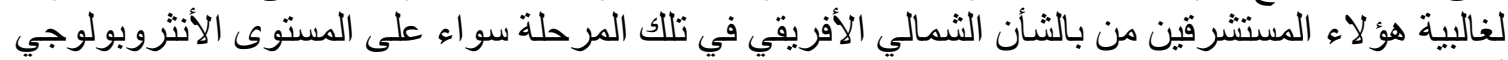

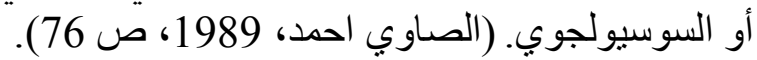

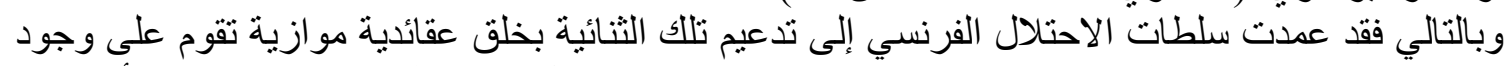

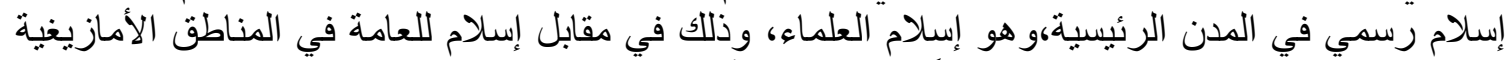

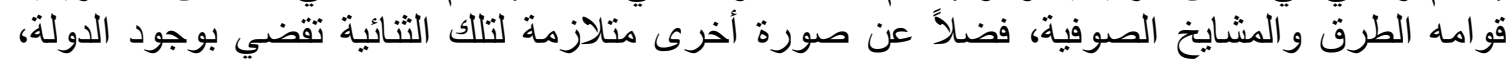

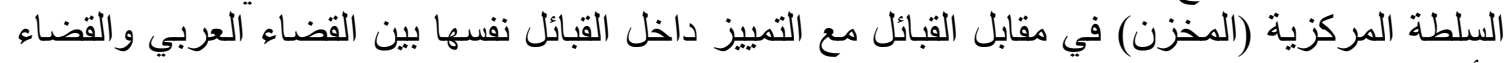

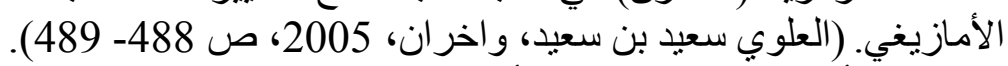

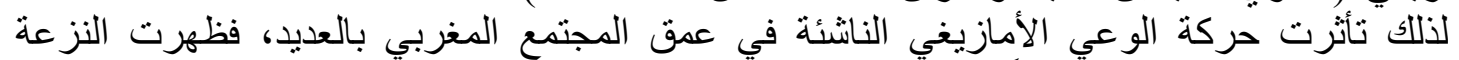

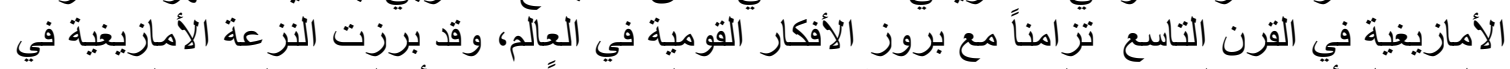

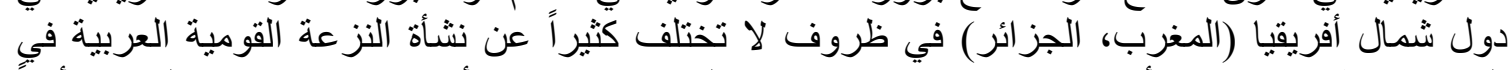

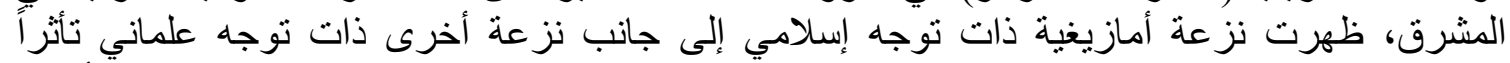

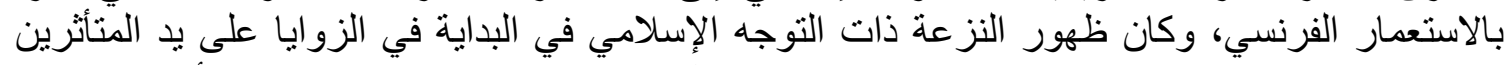

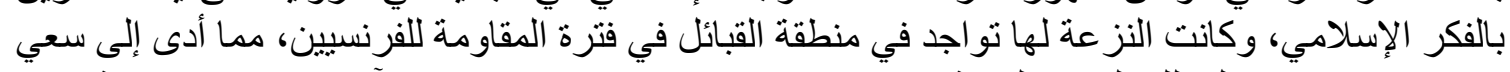

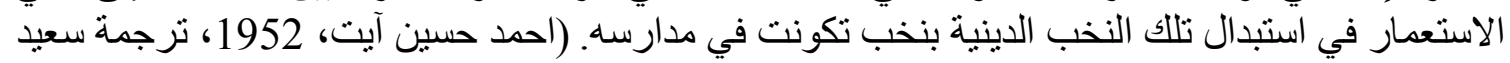

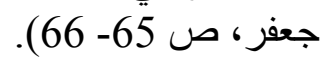

\section{الأزمة الأمازيغية}

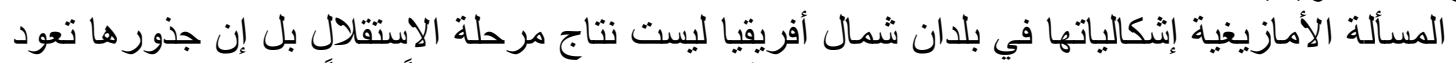

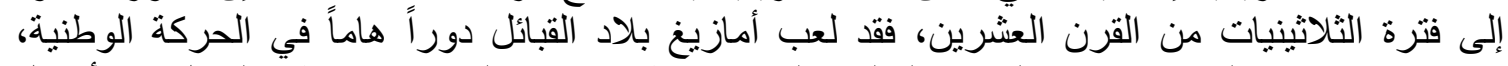

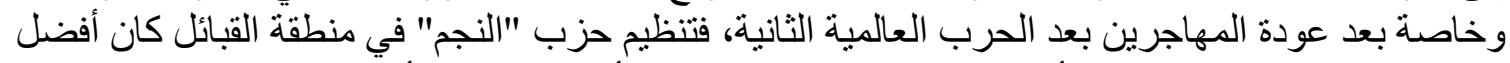

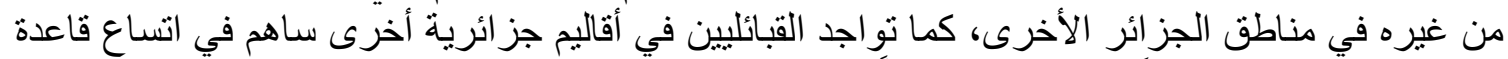

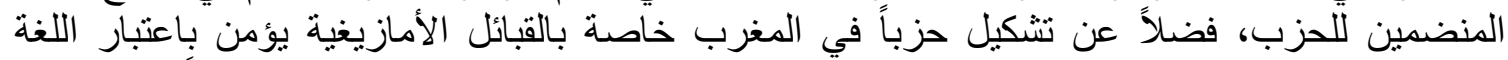

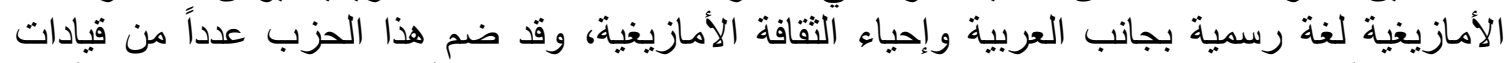

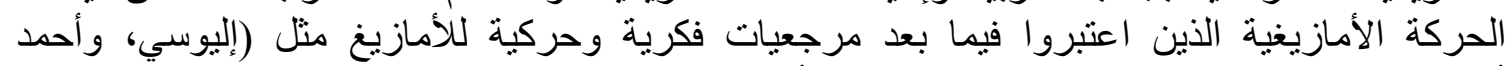

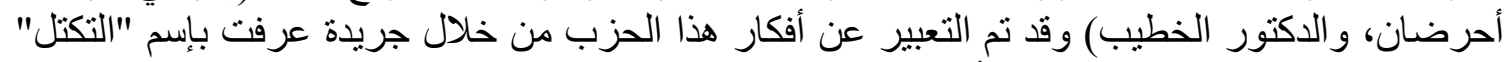

التي كانت تطالب بضرورة تدريس الأمازيغية في المدارس المغربية. (Anna Morath, 2012 ( p.30 ). 


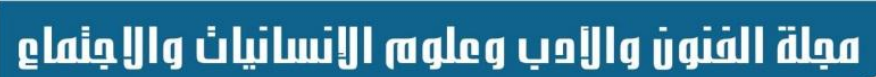 Journal of Arts, Literature, Humanities \\ and Social Sciences}

ISSN print: 2616- 3810

ISSN online: 2414 - 3383
Volume 48

January 2020
LALLHSS

www.jalhss.com

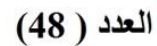

يناير 2020

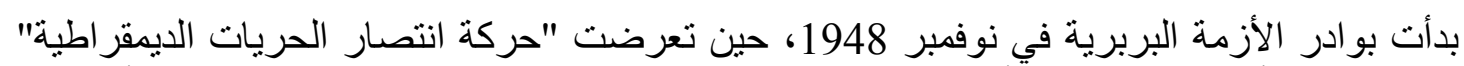

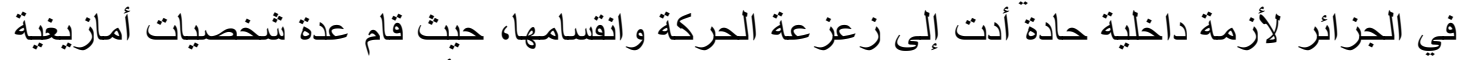

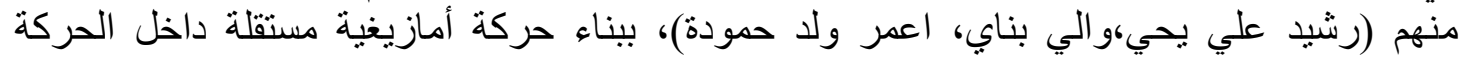

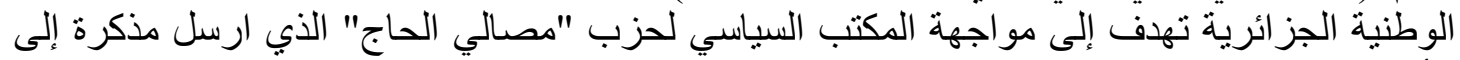

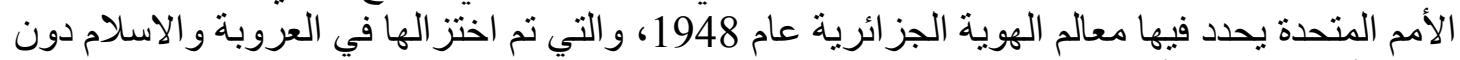

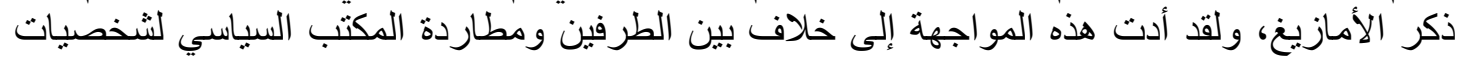

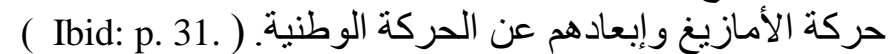

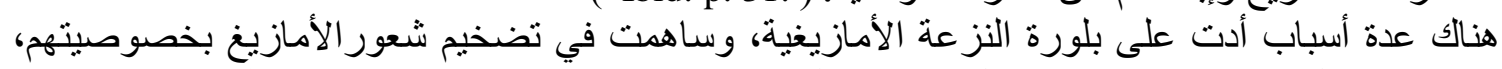

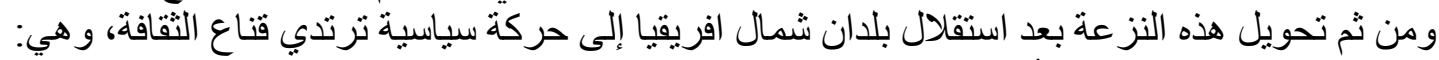
1- انغلاق المنطقة الريفية الأمازيغية على نفئل نفسها.

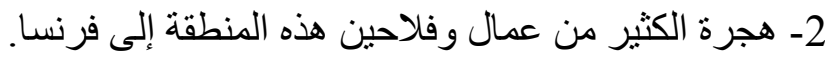

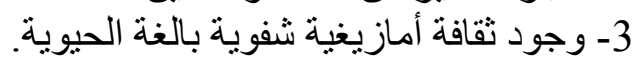

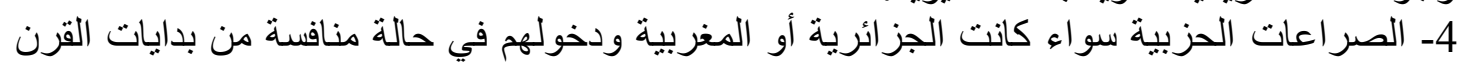

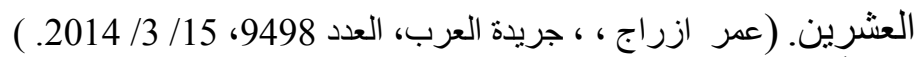

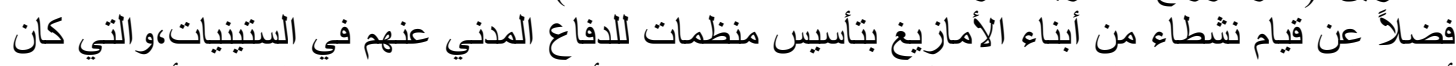

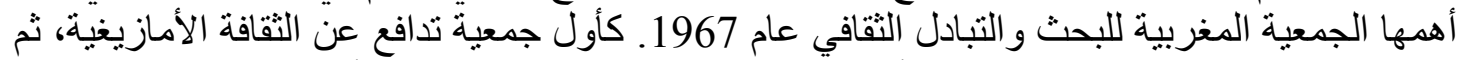

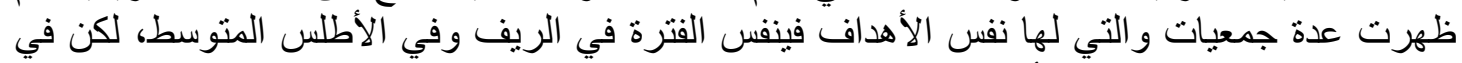

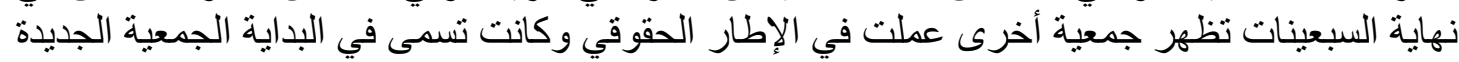

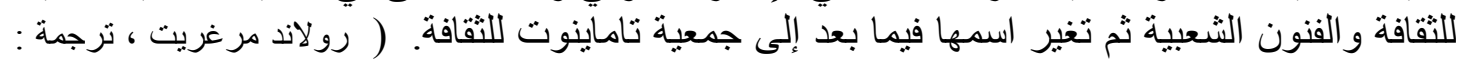

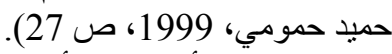

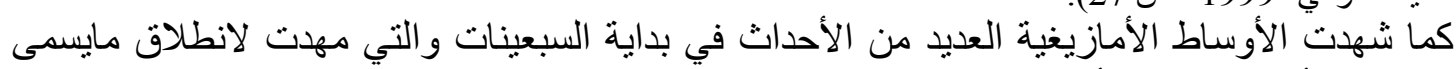

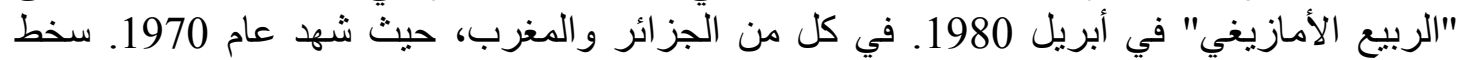

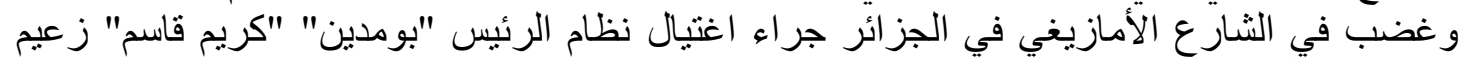

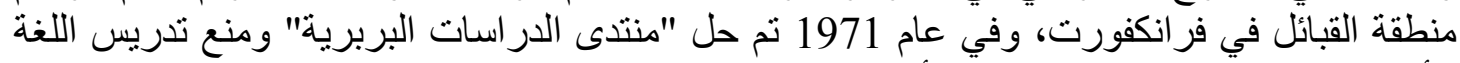

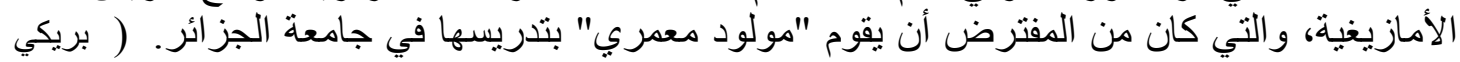
محمد عاطف ، 2012.

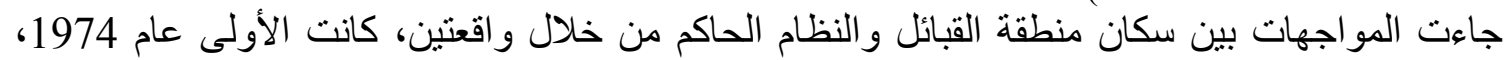

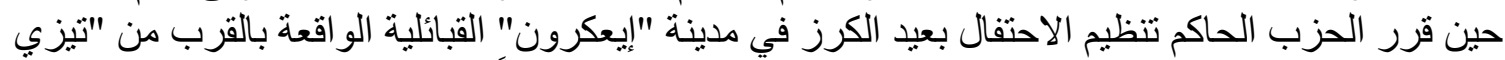

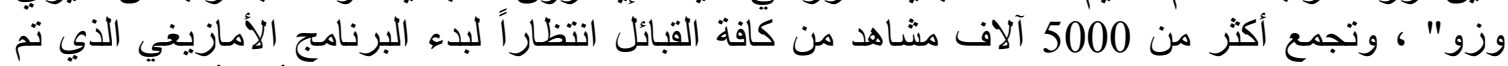

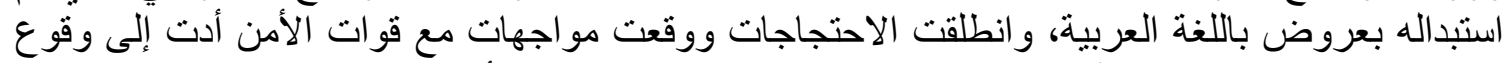

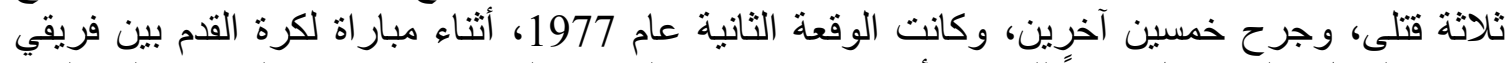

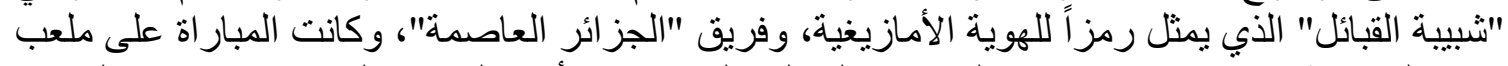

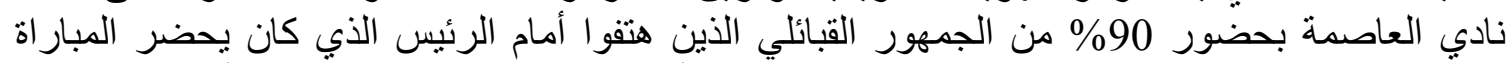

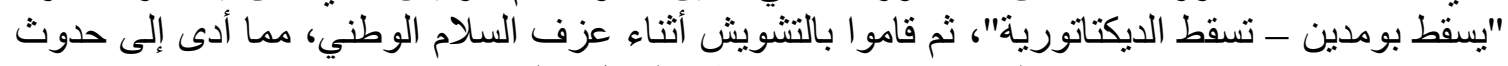

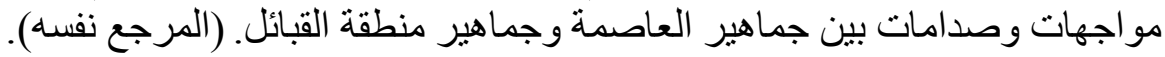

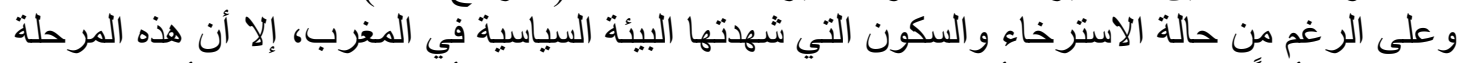

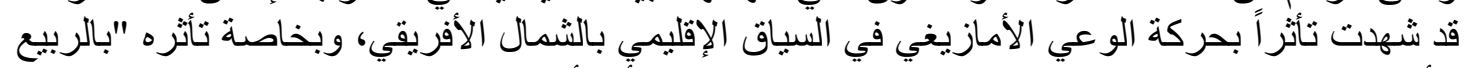

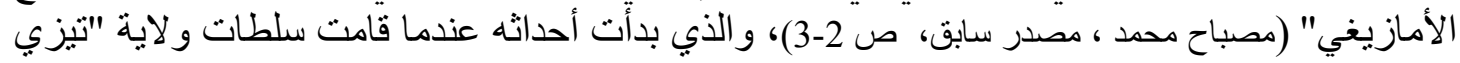

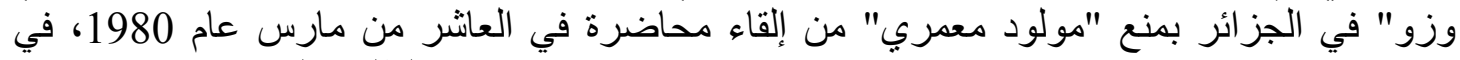

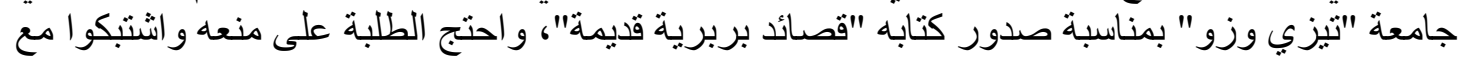




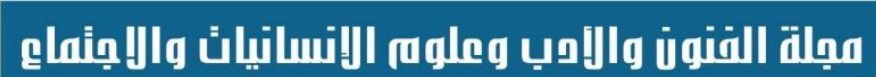 Journal of Arts, Literature, Humanities \\ and Social Sciences}

ISSN print: 2616- 3810

ISSN online: 2414 - 3383
Volume 48

January 2020
¿ÁLHSS

www.jalhss.com

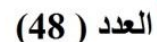

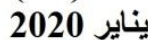

قوات مكافحة الثخب والجيش لمدة أسبوعين متتاليين، وتحول الاحتجاج ليشمل كافة فئات المجتمع

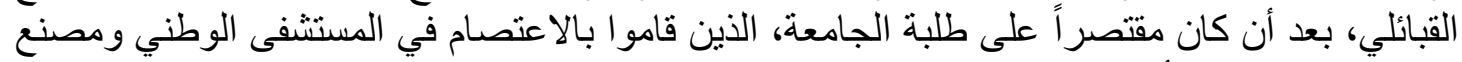

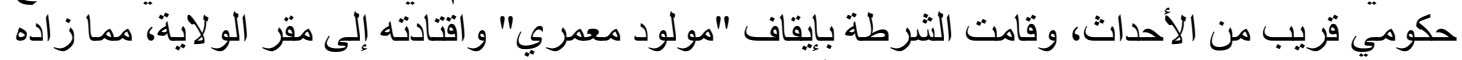

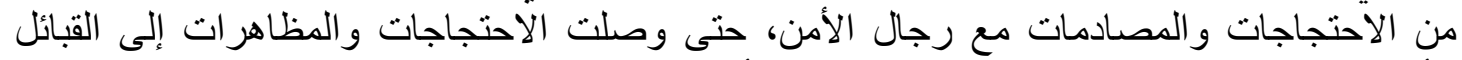

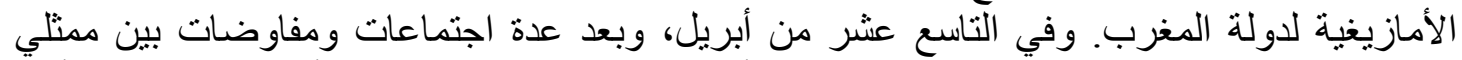

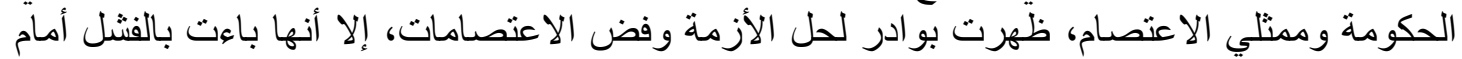

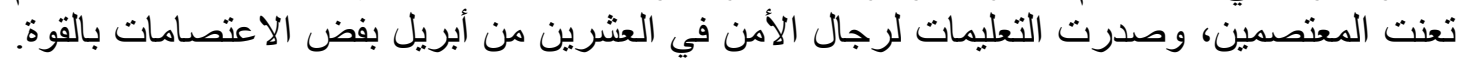
(بريكي محمد عاطف، مصدر سابق، الصفحة نفسها).

تداعيات أحداث ابريل 1980 الربيع الأمازيغي على القضية الأمازيغية

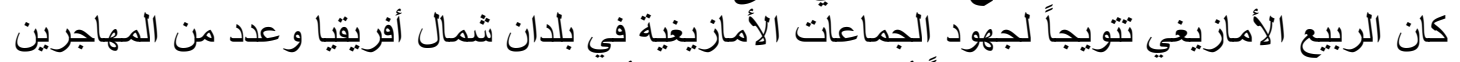

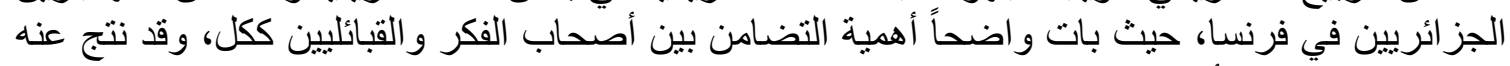
العديد من التداعيات أهمها: 1- كان هدف الربيع الأمازيغي إثغاله القضية وكسر حاجز الصمت الصنات عن قضية الأمازيغ في شمال أفريقيا،

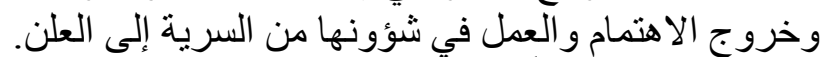

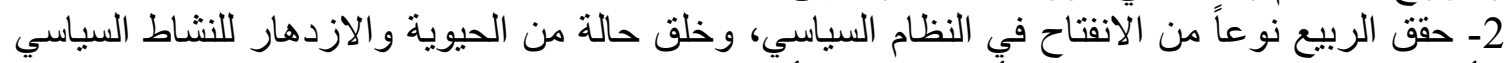

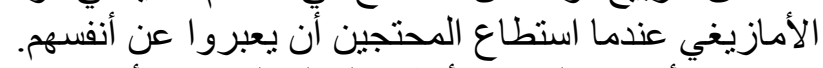

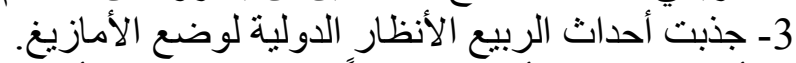

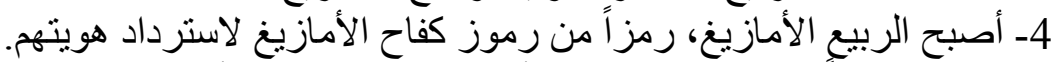

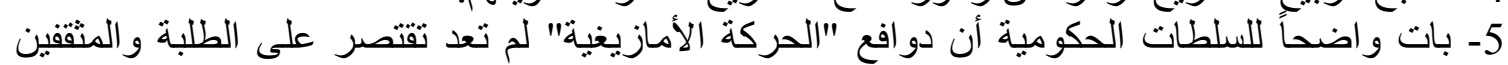

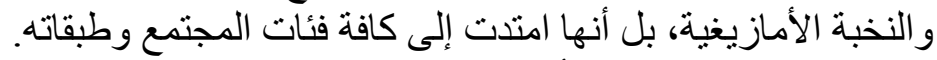

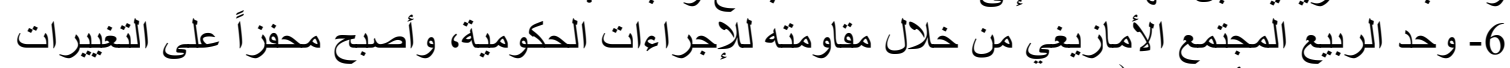

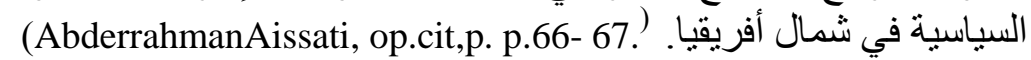

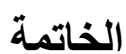

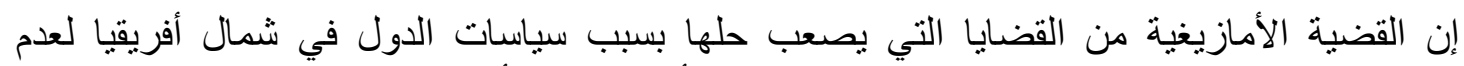

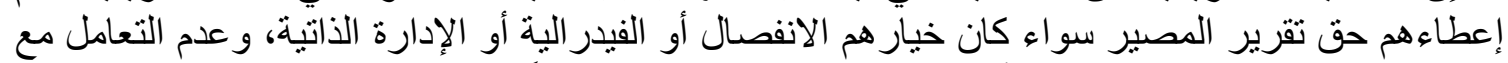

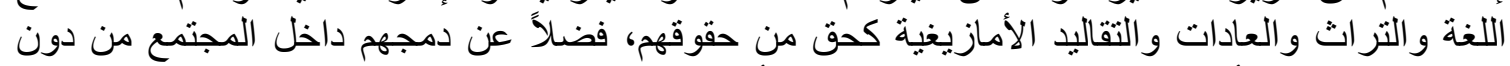

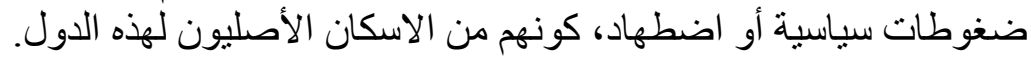

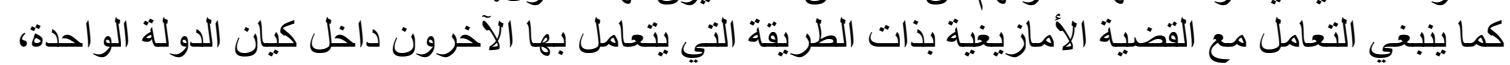

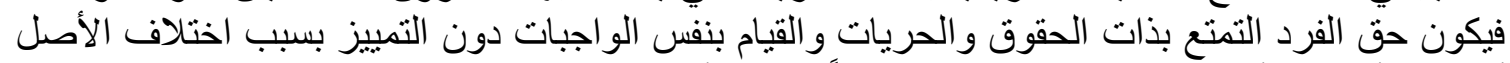

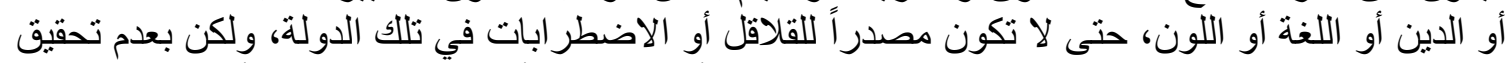

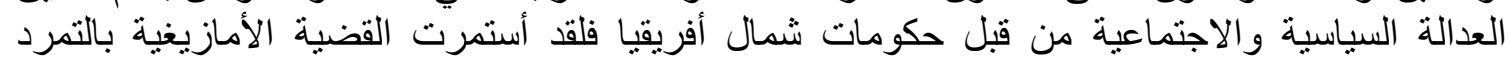

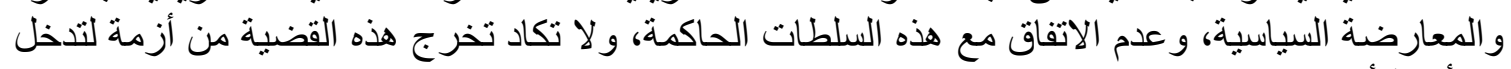
في أزمة أخرى جديدة.

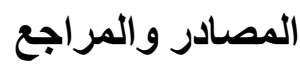




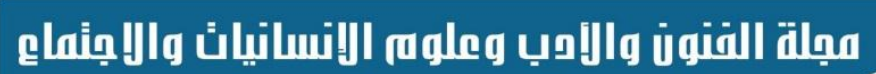 Journal of Arts, Literature, Humanities \\ and Social Sciences}

ISSN print: 2616- 3810

ISSN online: 2414 - 3383
Volume 48

January 2020
LALLHSS

www.jalhss.com

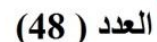

يناير 2020

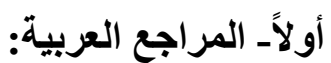

(1) الصاوي أحمد. (1989). الأقليات التاريخية في الوطن العربي،(ط 1)، القاهرة- مصر : مركز الحضارة العربية للإعلام و النشر. (2) الدر اجي بوزياني .(2003). القبائل الأمازيغية أدوارها ـ مواطنها ـ أعيانها، (ط 1)، الجز ائر ـ الجزائر: دار

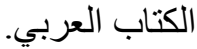
(3) الحمدو اي جميل. (2015). الحضارة الأمازيغية انثروبولوجية التاريخ ـ الكتابة، الديانات، والثقافة ، المغرب، الدار البيضـاء: دار افريقيا الثرق. (2015.

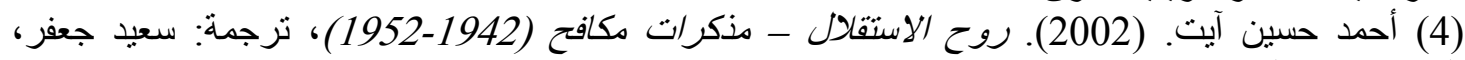
الجزائر: دار البرزخ.

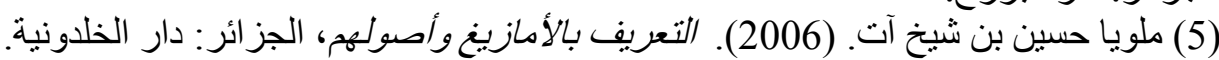

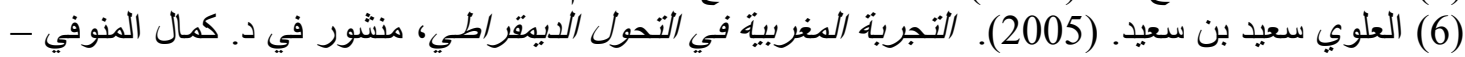

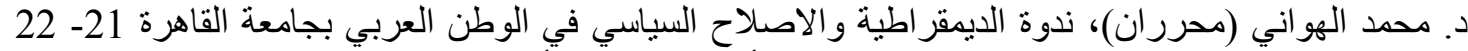

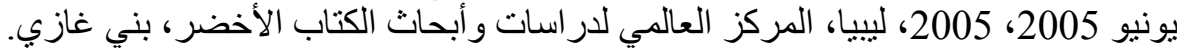

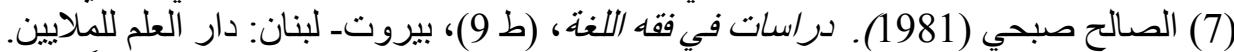

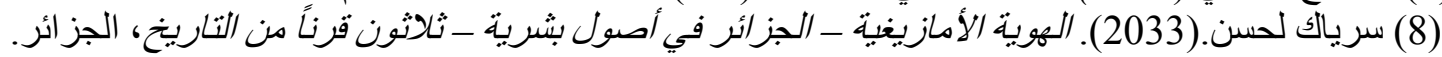
(10) مادون محمد علي. (1992). عروبة البربر - الحقبقة الدغدورة، دمشق: المنظمة العربية للتربية و الثقافة التية و العلوم. الطباعة. (12) مصباح محمد. (2011م). الأمازيغية في الدغرب - جدل الداخل والخارج، الدوحة ، قطر : مركز العربي للابحاث.

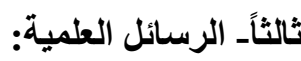

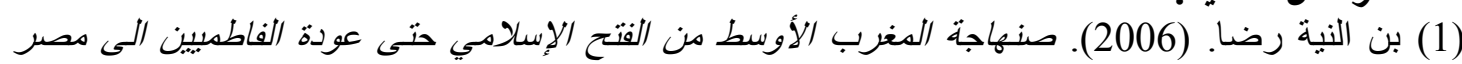

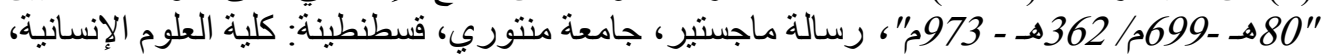

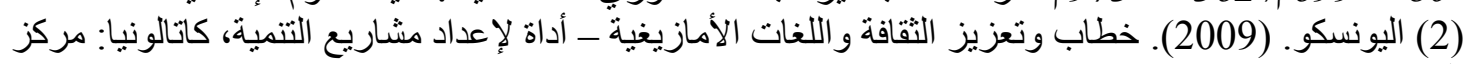

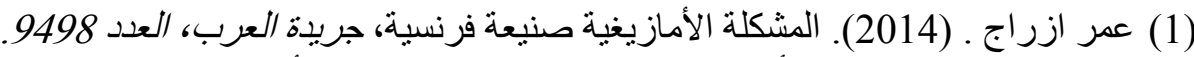

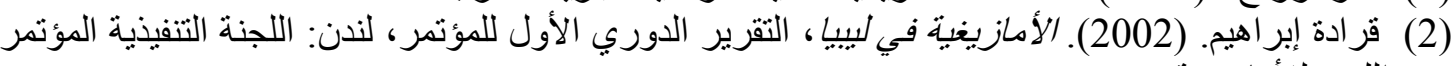
الليبي للأمازيغية. (3) محمد عاطف بريكي. (2012). بعد 32 سنة على مروره - هل فقد الربيع الأمازيغي عنفوانه، جريبة

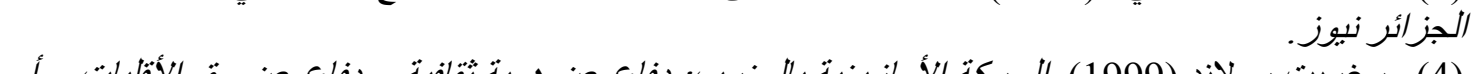

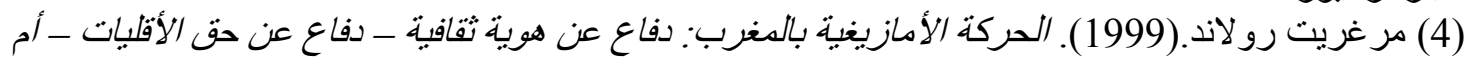

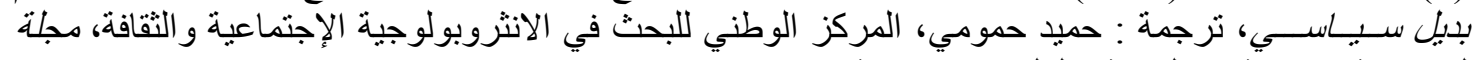
الجزائرية في الانثروبولوبية ترجة والعلوم الاجتماعية.

\section{References}

1. Al-Sawy Ahmed. (1989). Historical Minorities in the Arab World, ( ${ }^{\text {st }}$ edition), 


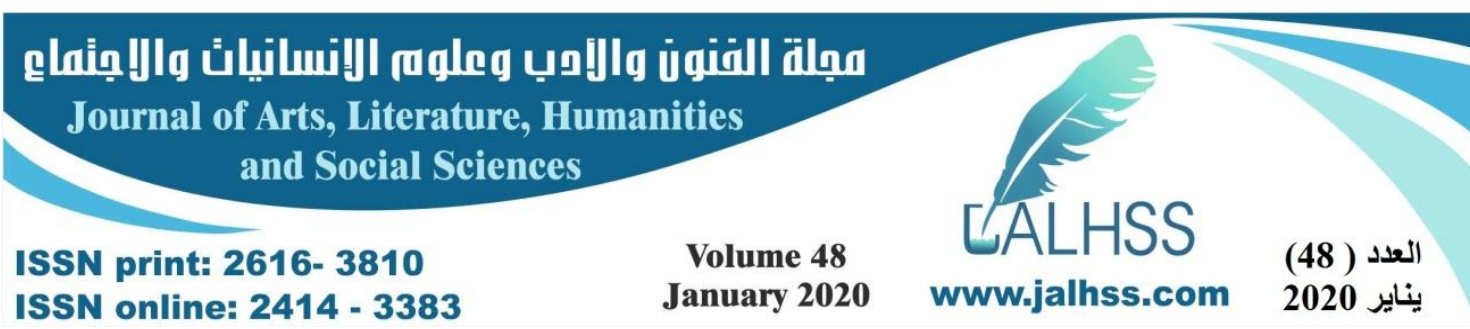

Cairo, Egypt: Arab Civilization Center for Media and Publishing.

2. Darraji Bouziani. (2003). Amazigh tribes: Their roles - their citizens - their elders, ( $1^{\text {st }}$ edition). Algeria - Algeria: Dar Al-Kitab Al-Arabi.

3. Praise be to God. (2015). The Amazigh Civilization Anthropology of History Writing, Religions, and Culture. Morocco, Casablanca: House of East Africa.

4. Ahmed Hussein Ait. (2002). Spirit of Independence - Memoirs of a Struggling (1942-1952), translation: Sa id Ja`far. Algeria: Dar al-Barzakh.

5. Malloya Hussein bin Sheikh is coming. (2006). Introducing the Berbers and their origins. Algeria: Dar Khaldounieh.

6. Al-Alawi Saeed bin Saeed. (2005). Moroccan experience in democratic transformation. published in d. Kamal Al-Menoufy - Dr. Muhammad Al-Hawani (eds.), Symposium on Democracy and Political Reform in the Arab World, Cairo University, 21-22 June 2005, 2005, Libya, International Center for Studies and Research on the Green Book, Bani Ghazi.

7. Al-Saleh Subhi (1981). Studies in jurisprudence, $\left(9^{\text {th }}\right.$ edition). Beirut - Lebanon: House of Knowledge for millions.

8. Seryak to Hassan. (2033). The Berber identity - Algeria in human origins - thirty centuries of history. Algeria.

9. Madun Muhammad Ali. (1992). Arabism of the Berbers - The Flooded Truth. Damascus: Arab Organization for Education, Culture and Science.

10. Al-Arabawi Mohammed Mukhtar. (2000). At the Roots of the National Question Ancient Arab Berbers, (2 ${ }^{\text {nd }}$ ed.).Tunisia: The Art of Printing.

11. Mesbah Muhammad. (2011). The Tamazight in Morocco - Internal and External Controversy. Doha, Qatar: Arab Research Center.

12. Bin Al-Niyyah Redha. (2006). The Cranes of the Central Maghreb from the Islamic Conquest to the Return of the Fatimids to Egypt "80 AH - 699 CE / 362 AH $973 C E^{\prime \prime}$, Master Thesis, University of Mentouri, Constantine: College of Humanities, 13. UNESCO. (2009). Speech and promotion of Amazigh culture and languages - a tool for preparing development projects, Catalonia: UNESCO Center.

14. Omar Azraj. (2014). The Berber problem is French workmanship. Al-Arab newspaper. issue 9498.

15. Ibrahim's tick. (2002). Tamazight in Libya. the first periodic report of the conference, London: The Executive Committee of the Libyan Berber Conference.

16. Mohamed Atef Braiki. (2012). 32 years after his passage - did the Amazigh spring lose its vigor. the Algeria News.

17. Margaret Roland. (1999). The Amazigh movement in Morocco: a defense of a cultural identity - a defense of the right of minorities - or a political alternative, translation. Hamid Hamoumi, the National Center for Research in Social Anthropology and Culture, Algerian Journal of Anthropology and Social Sciences. 